\title{
Eravacycline for treatment of complicated intra-abdominal infections: the fire is not ignited!
}

\author{
Patrick M. Honore, Herbert D. Spapen \\ Intensive Care Medicine, ICU Department, Universitair Ziekenhuis Brussel, Vrije Universiteit Brussel, Brussels, Belgium \\ Correspondence to: Prof. Patrick M. Honore, MD, PhD, FCCM. Intensive Care Medicine, ICU Department, Universitair Ziekenhuis Brussel, Vrije \\ Universiteit Brussel, 101, Laarbeeklaan, 1090 Jette (Brussels), Brussels, Belgium. Email: Patrick.Honore@az.vub.ac.be. \\ Provenance: This is a Guest Editorial commissioned by Section Editor Mi Zhou, MM (No. 152 Central Hospital of PLA, Pingdingshan, China). \\ Comment on: Solomkin J, Evans D, Slepavicius A, et al. Assessing the Efficacy and Safety of Eravacycline vs Ertapenem in Complicated Intra- \\ abdominal Infections in the Investigating Gram-Negative Infections Treated With Eravacycline (IGNITE 1) Trial: A Randomized Clinical Trial. \\ JAMA Surg 2017;152:224-32.
}

Submitted Jul 22, 2017. Accepted for publication Jul 31, 2017.

doi: $10.21037 /$ atm.2017.08.15

View this article at: http://dx.doi.org/10.21037/atm.2017.08.15

The worldwide surge of multi-drug resistant (MDR) Gramnegative infections has become a real threat in postsurgical and critically ill patients. Among the most dreaded MDR Gram-negatives are extended-spectrum $\beta$-lactamase (ESBL) and carbapenemase producing Enterobacteriaceae and MDR Pseudomonas species $(1,2)$. Carbapenem-resistant Enterobacteriaceae (CRE), in particular, are a steadily growing plague associated with increased morbidity and high mortality rates (3). Against this MDR Gram-negative epidemic stands a long period of antibiotic "starvation", interrupted only by the market introduction of tigecycline and doripenem (4). This often left clinicians with no other option than choosing for "older" polymyxins and/ or aminoglycosides as primary treatment of complicated urinary, abdominal, pulmonary, and blood infections caused by MDR Gram-negative bacteria $(5,6)$. Unfortunately, these two antibiotic classes have no well-defined or an unpredictable pharmacokinetic and pharmacodynamic profile which hampers their efficacy whilst enhancing the risk for adverse effects and toxicity.

Partial relief from this distressing situation was provided by joint initiatives of national healthcare agencies, governmental institutions, and the pharmaceutical industry, to reinvigorate the development of new potent antibiotics. Combinations of either an existing $\beta$-lactam with a novel $\beta$-lactamase inhibitor (ceftazidime-avibactam) (7) or vice versa (ceftolozane-tazobactam) already emerged from this pipeline (8).

Eravacycline is a novel fluorocycline antibiotic that has reached phase 3 study evaluation. Basically, eravacycline has a modified tetracycline core structure which enables to overcome tetracycline-specific resistance mechanisms such as efflux pumps and ribosomal hydrolysis (9) but also renders it 2- to 4-fold more potent in vitro than tigecycline against CRE (10). Eravacycline showed important activity against MDR Gram-positive and Gram-negative microorganisms, including those expressing ESBL or carbapenem resistance mechanisms $(11,12)$.

In a recent issue of $\mathcal{F} A M A$ Surgery, Solomkin et al. presented the results of the IGNITE 1 trial assessing efficacy and safety of eravacycline vs. ertapenem in complicated intra-abdominal infections (cIAIs). This large phase 3 study enrolled 541 patients who were randomized to receive either intravenous (IV) eravacycline $(1.0 \mathrm{mg} / \mathrm{kg} / 12 \mathrm{~h}$; 270 patients) or IV ertapenem (1.0 g daily; 271 patients). At the test-of-cure visit in the microbiologically intent-to-treat population (446 subjects), the clinical cure rates were $86.8 \%$ and $87.6 \%$ respectively demonstrating non-inferiority of eravacycline to ertapenem (13).

Despite presenting treatment groups with very wellbalanced patient characteristics, surgical techniques, and infection types at baseline, the IGNITE 1 trial is far from confirming a potential benefit of eravacycline in this particular indication. First, the investigators took care to include only severe cIAIs, yet the low mean acute physiology and chronic health evaluation (APACHE) II scores in both groups refer to a low global disease severity with a predicted hospital mortality between 0 and $5 \%(14,15)$. This is mainly 
driven by the exclusion of more severely ill patients, in particular those with septic shock and acute kidney injury. Second, the number of CREs (18 in each group) is too low to positively evaluate a potential therapeutic superiority of eravacycline against these micro-organisms. Third, the less favorable response of Enterococcus faecalis to eravacycline is somewhat cumbersome for a drug with assumed good activity against MDR Gram-positive bacteria, including vancomycin-resistant enterococci. In contrast, eravacycline provided clinical cure in more than $80 \%$ of patients infected with Pseudomonas aeruginosa despite its known poor in vitro activity against this bacterium. This may be due to a favorable reaction on even a short anti-Pseudomonal $\beta$-lactam use in the perioperative phase in association with a rapid and adequate surgical source control. Finally, patients treated with eravacycline were more likely to have one of the particular well-defined reasons of clinical failure. In the margin of the current trial, it is notably that a phase 3 clinical trial comparing eravacycline with levofloxacin for the treatment of complicated urinary tract infections did not achieve its primary endpoint of non-inferiority (16).

Taken together, the IGNITE 1 study does not offer convincing proof to propose eravacycline as a first-line drug for the treatment of cIAIs. It clearly does not challenge a standard carbapenem for treating ESBL-positive strains and CREs and probably will never rival with the novel $\beta$-lactam/ $\beta$-lactamase combinations in a MDR Pseudomonas environment. Moreover, its efficacy in more severely ill and consequently more-difficult-to treat patients remains to be established.

\section{Acknowledgements}

None.

\section{Footnote}

Conflicts of Interest: The authors have no conflicts of interest to declare.

\section{References}

1. Thaden JT, Pogue JM, Kaye KS. Role of newer and reemerging older agents in the treatment of infections caused by carbapenem-resistant Enterobacteriaceae. Virulence 2017;8:403-16.

2. Hawkey PM. Multidrug-resistant Gram-negative bacteria: a product of globalization. J Hosp Infect 2015;89:241-7.
3. Tzouvelekis LS, Markogiannakis A, Psichogiou M, et al. Carbapenemases in Klebsiella pneumoniae and other Enterobacteriaceae: an evolving crisis of global dimensions. Clin Microbiol Rev 2012;25:682-707.

4. Falagas ME, Mavroudis AD, Vardakas KZ. The antibiotic pipeline for multi-drug resistant gram negative bacteria: what can we expect? Expert Rev Anti Infect Ther 2016;14:747-63.

5. Honoré PM, Jacobs R, Joannes-Boyau $\mathrm{O}$, et al. Continuous renal replacement therapy-related strategies to avoid colistin toxicity: a clinically orientated review. Blood Purif 2014;37:291-5.

6. Brasseur A, Hites M, Roisin S, et al. A high-dose aminoglycoside regimen combined with renal replacement therapy for the treatment of MDR pathogens: a proof-ofconcept study. J Antimicrob Chemother 2016;71:1386-94.

7. Mazuski JE, Gasink LB, Armstrong J, et al. Efficacy and Safety of Ceftazidime-Avibactam Plus Metronidazole Versus Meropenem in the Treatment of Complicated Intra-abdominal Infection: Results From a Randomized, Controlled, Double-Blind, Phase 3 Program. Clin Infect Dis 2016;62:1380-9.

8. Solomkin J, Hershberger E, Miller B, et al. Ceftolozane/ Tazobactam Plus Metronidazole for Complicated Intraabdominal Infections in an Era of Multidrug Resistance: Results From a Randomized, Double-Blind, Phase 3 Trial (ASPECT-cIAI). Clin Infect Dis 2015;60:1462-71.

9. Zhanel GG, Cheung D, Adam H, et al. Review of Eravacycline, a Novel Fluorocycline Antibacterial Agent. Drugs 2016;76:567-88.

10. Livermore DM, Mushtaq S, Warner M, et al. In Vitro Activity of Eravacycline against Carbapenem-Resistant Enterobacteriaceae and Acinetobacter baumannii. Antimicrob Agents Chemother 2016;60:3840-4.

11. Sutcliffe JA, O'Brien W, Fyfe C, et al. Antibacterial activity of eravacycline (TP-434), a novel fluorocycline, against hospital and community pathogens. Antimicrob Agents Chemother 2013;57:5548-58.

12. Monogue ML, Thabit AK, Hamada Y, et al. Antibacterial Efficacy of Eravacycline In Vivo against Gram-Positive and Gram-Negative Organisms. Antimicrob Agents Chemother 2016;60:5001-5.

13. Solomkin J, Evans D, Slepavicius A, et al. Assessing the Efficacy and Safety of Eravacycline vs Ertapenem in Complicated Intra-abdominal Infections in the Investigating Gram-Negative Infections Treated With Eravacycline (IGNITE 1) Trial: A Randomized Clinical Trial. JAMA Surg 2017;152:224-32. 
14. Nag DS. Assessing the risk: Scoring systems for outcome prediction in emergency laparotomies. Biomedicine (Taipei) 2015;5:20.

15. Agarwal A, Choudhary GS, Bairwa M, et al. Apache II scoring in predicting surgical outcome in patients of

Cite this article as: Honore PM, Spapen HD. Eravacycline for treatment of complicated intra-abdominal infections: the fire is not ignited! Ann Transl Med 2017;5(21):425. doi: 10.21037/ atm.2017.08.15 perforation peritonitis. Int Surg J 2017;4:2321-5.

16. Tetraphase announces top-line results from IGNITE2 phase 3 clinical trial of eravacycline in cUTI.

Available online: http://ir.tphase.com/releasedetail. cfm? releaseid $=930613$ 